\title{
CONTAMINACIÓN ATMOSFÉRICA Y EFECTOS RESPIRATORIOS EN NIÑOS, EN MUJERES EMBARAZADAS Y EN ADULTOS MAYORES
}

\section{POLLUTION AND RESPIRATORY EFFECTS ON CHILDREN, PREGNANT WOMEN AND OLD AGED PEOPLE}

\author{
Sandra Vargas ${ }^{1}$ \\ William Onatra ${ }^{2}$ \\ Lucia Osorno ${ }^{3}$ \\ Eduardo Páez ${ }^{3}$ \\ Orlando Sáenz $z^{4}$
}

\section{RESUMEN}

Las enfermedades respiratorias como la infección respiratoria aguda (IRA) y la enfermedad pulmonar obstructiva crónica (EPOC).son conjunto de patologías que afectan el sistema respiratorio, siendo causa frecuente de morbilidad y mortalidad en los niños y niñas menores de cinco años, mujeres embarazadas y adultos mayores. Las fuentes principales de contaminación de origen humano son las quemas de desechos en el área rural, minería y canteras, manufactura, industria química, refinería de petróleo, generadores de energía, motores de combustión e incineradores, el transporte urbano, la producción de energía y las actividades industriales. Todas ellas se acumulan en las ciudades o en sus alrededores por lo que provocan una gran contaminación del aire exterior. Los contaminantes del aire son partículas sólidas en suspensión (PSS), gases

\footnotetext{
${ }^{1}$ MD. M.Sc. Salud Pública. Docente Carrera de Medicina, Universidad de Ciencias Aplicadas y Ambientales U.D.C.A. Dirección para correspondencia: Calle 222 No.55-37.svargas@udca.edu.co

2 MD. Ginecólogo-Endocrinólogo. Especialista en Biología Reproductiva. Ginecólogo-Endocrinólogo. Docente de Ginecología y Obstetricia, Carrera de Medicina, U.D.C.A

${ }^{3}$ MD. Docente de Medicina U.D.C.A

${ }^{4}$ Vicerrector de Investigaciones U.D.C.A hasta 2006.
}

y olores. Recientemente se ha prestado atención a la proporción de PSS con tamaño inferiores a $10 \mu \mathrm{m}$ (Material Particulado PM10) que pueden ser inhaladas y penetrar al pulmón causando efectos nocivos para la salud. Se correlacionan los efectos de los principales contaminantes inorgánicos gaseosos como el dióxido de azufre (( $\left.\mathrm{SO}_{2}\right)$, el monóxido de carbono (CO), dióxido de nitrógeno $\left(\mathrm{NO}_{2}\right)$, algunos hidrocarburos $(\mathrm{HC})$ como el benceno, butadieno y los efectos a nivel pulmonar en tres grupos poblacionales niños menores de cinco años, embarazo y adulto mayor.

Palabras clave: Enfermedad respiratoria aguda, contaminación ambiental, aire.

\section{SUMMARY}

Respiratory diseases as acute respiratory infection (ARI) and pulmonary chronic obstructive disease (PCOD) are a group of pathologies that affect the respiratory system, being a very common cause of morbidity and mortality on children under five years, pregnant women and adult elders. The main sources of contamination are burning remainders in the rural area, mining and quarries, manufactures, chemical industry, petroleum refinery, generators, motors of combustion and incinerators, the urban transport, the industrial production of energy and other industrial activities All of them are accumulated around in the cities causing a great pollution of the out air. The polluting agents are solid particles in suspension in the air 
(PSS), gases and scents. In the last years, attention has been paid to the proportion of PSS with inferior sizes to $10 \mu \mathrm{m}$ (particulated material PM 10) that can be inhaled and break into the lung causing injurious effects for the health. Also, there are correlation with the principal inorganic gases compounds like sulfur dioxide (SO2), the carbon monoxide ( $\mathrm{CO}$ ) and the secondary nitrogen dioxide (NO2), as well as the some hydrocarbons ( $\mathrm{HC})$ as benzene, butadiene and effects on pulmonary disease in three population groups: children under five years. pregnant women and adult elders.

Key words: Acute respiratory disease, ambient contamination, air.

\section{INTRODUCCIÓN}

En el Enfoque Ecosistémico en Salud Humana, el ambiente urbano se caracteriza por ser un ecosistema altamente influenciado por la actividad humana. Sus características distintivas son la elevada densidad de población, la infraestructura establecida y el alto nivel de organización social. Las ciudades presentan desafíos específicos para el enfoque de la salud y el medio ambiente. Reflexionar sobre el hecho urbano en su perspectiva ambiental conduce necesariamente a entender la dinámica relacional de un sistema complejo asignado por determinaciones culturales, donde el ser humano, en sus procesos de adaptación tecnológica, regula el equilibrio de los nuevos sistemas que construye. Si bien, la ciudad como creación humana pertenece al campo de la cultura, su comprensión ambiental sólo se podrá dar en la medida en que se analice en su relación con el entorno natural que la sustenta (Krewki E Rainham, 2007; Fondo Financiero Distrital, 1999).

La Cumbre de la Tierra, celebrada en Río de Janeiro, en 1992, subrayó que el desarrollo significa cubrir las necesidades de las personas, su salud, su bienestar, su vida y el medio ambiente (OPS, 2000). La calidad del ambiente es fundamental para la salud humana. Para ello es importante describir los efectos nocivos de la contaminación y la degradación ambiental y demostrar que un medio ambiente satisfactorio permite la salud y el desarrollo. La Organización Mundial de la salud (OMS) calcula que 13 millones de niños y niñas menores de cinco años mueren cada año en el mundo y el $99 \%$ de éstas, en el 2000, sucederán en países en vía de desarrollo (WHO, 1997).
El deterioro del medio ambiente ha sido identificado en Colombia y en el mundo como uno de los problemas más serios que afronta la humanidad. Es un fenómeno relativo que depende de muchas variables. Los problemas generados por el rápido y desordenado proceso de urbanización de Bogotá, las condiciones sanitarias, la marginalidad, la inequidad, los factores demográficos, ambientales, socioeconómicos y los estilos de vida nocivos son determinantes de primer orden en el proceso salud-enfermedad de la población capitalina (Secretaría Distrital de Salud, 2004).

La mala calidad del medio ambiente puede traer consigo mayores niveles de exposición y riesgo, así como la forma en que distintos ambientes degradados se asocian a determinados efectos en la salud. La degradación de la calidad del medio puede causar exposiciones nocivas para el hombre, con posibles efectos para su salud. Sin embargo, la magnitud de estas exposiciones depende no solo de su nivel, sino también de la proximidad de la población a la amenaza. Por consiguiente, cantidades relativamente pequeñas de contaminantes pueden tener un gran impacto si se liberan cerca de asentamientos humanos (Viegi et al. 2006). La contaminación del aire, especialmente en ciudades, ha sido un área de especial atención por las directivas de salud y sus entes gobernantes. Desde la década de 1950, se ha acumulado un conjunto de evidencias que muestran que la contaminación del aire urbano tiene efectos nocivos sobre la salud (Pope et al. 2002). Así es como actualmente se estima, a nivel mundial, que una cantidad muy grande, del orden de mil quinientos millones de individuos, está expuesta a niveles altos de contaminantes en el aire; que entre 20 y $30 \%$ de las enfermedades respiratorias de todo tipo parecen ser causadas por la contaminación del aire en interiores y en exteriores; que de 4 a $8 \%$ de las muertes prematuras, se deben a partículas en el aire interior y exterior; que cerca de 500 mil muertes anuales, se atribuyen a la contaminación del aire exterior, con partículas y dióxido de azufre y que dos millones de muertes anuales, se deben a la exposición a altas concentraciones de partículas en el aire de interiores (CEPIS -OPS, 2006, Osmond \& Baker, 2000).

Los efectos de los contaminantes del aire en la salud, se deben efectuar en forma integral tomando en cuenta la participación y la ponderación de la contaminación, tanto en interiores como de exteriores. Los niveles de contaminación del aire han sido ampliamente estudiados 
en algunas regiones del planeta, mientras que sus efectos y su impacto en la salud están debidamente descritos (Dockery, 1994; Heinrich et al. 1999).

El estudio y análisis de riesgos en el mundo actual plantea la construcción de un marco conceptual y metodológico propio que conlleva a la articulación de elementos capaces de interpretar las relaciones entre naturaleza y sociedad. El desafío consiste en que las instituciones locales puedan conocer, en su propia realidad, los niveles de contaminación y las condiciones de exposición de sus poblaciones, para definir la magnitud del riesgo que enfrentan y para establecer, con criterios objetivos, sus normas de calidad del aire. A ello contribuye la divulgación de la información relativa a cómo evaluar los riesgos (National Children's Study, 2004).

\section{CONTAMINACIÓN DEL AIRE}

La contaminación del aire es un problema que afecta por igual a los países desarrollados y en desarrollo. Sus efectos en la salud son cuestiones muy complejas, ya que son muchos los contaminantes existentes, por consiguiente, es difícil discernir el efecto de cada uno del de los demás. Se sabe, sin embargo, que la contaminación del aire influye desfavorablemente en la población expuesta. Los contaminantes inhalados afectan el aparato respiratorio, pasan a la sangre y alcanzan a todos los órganos (Sram et al. 2005). Además, se depositan en el agua, en las plantas y en el suelo contribuyendo a la exposición humana a través de la ingestión de alimentos y agua contaminados. Las partículas sólidas en suspensión en el aire (PSS) afectan a la población mundial de forma más permanente que cualquier otro contaminante; se dispone de datos sobre las concentraciones de partículas en la atmósfera, datos epidemiológicos sobre su exposición y efectos en la salud, más que de cualquier otro contaminante (OPS, 2000). Sin embargo, la presencia de otros contaminantes, se halla íntimamente asociada a la de partículas y éstos contribuyen al deterioro de la salud (Kettunen et al. 2007). Algunos efectos específicos en la salud, como la enfermedad cardiovascular debida al monóxido de carbono (CO), se puede atribuir directamente a la exposición a contaminantes distintos a las PSS. (Schikowski et al. 2007).

Las fuentes principales de contaminación de origen humano son el transporte, la producción de energía y las actividades industriales, acumuladas en las ciudades o a su alrededor, por lo que provocan una gran contaminación del aire exterior. Los contaminantes del aire son PSS, gases y olores. Las PSS y los gases proceden de numerosas fuentes. En los últimos años, se ha prestado atención a la proporción de PSS como la PM con tamaños inferiores a 10 $\mu$ m (PM 10 y menores de $25 \mu \mathrm{m}$ (PM 2,5), que pueden ser inhaladas y penetrar en la profundidad del pulmón causando efectos nocivos para la salud (Romeo et al. 2006).

Las partículas tienen composiciones químicas y efectos en la salud muy diferentes dependiendo de su origen e interacción con otros componentes del aire. Por ejemplo, las partículas del plomo, se sabe que resultan especialmente peligrosas para los niños; otros componentes son motivo de preocupación, como el arsénico, el níquel, el cadmio y los presentes en los humos de los motores diesel (OPS, 2000; Salam et al. 2005).

También son dañinos para la salud los compuestos inorgánicos gaseosos, como el dióxido de azufre $\left(\mathrm{SO}_{2}\right)$, el monóxido de carbono (CO) y el dióxido de nitrógeno $\left(\mathrm{NO}_{2}\right)$, así como los hidrocarburos $(\mathrm{HC})$, como el benceno y el butadieno, otros compuestos orgánicos volátiles y los contaminantes secundarios (Tabla 1). Estos últimos, se deben a las reacciones químicas que tienen lugar en la atmósfera. Por ejemplo, el $\mathrm{SO}_{2}$, se puede oxidar y disolver en el agua formando nieblas de ácido sulfúrico. Las reacciones entre los óxidos de nitrógeno $\left(\mathrm{NO}_{2}\right)$ y los $\mathrm{HC}$ en presencia de la luz solar producen ozono $\left(\mathrm{O}_{3}\right)$, principal componente nocivo de los vapores fotoquímicos (CEPIS-OPS, 2006). Los mayores niveles de contaminación por $\mathrm{O}_{3}$ se registran en tiempo soleado, causando enfermedad pulmonar y asma en niños (OMS, 2006).

En los países desarrollados, el cuadro general muestra un descenso de las concentraciones de $\mathrm{SO}_{2}$ y PSS, con concentraciones de $20 \mu \mathrm{g} / \mathrm{m}^{3}$ media en 24 horas o $500 \mu \mathrm{g} / \mathrm{m}^{3}$ en $10 \mathrm{~min}$. Su exposición a largo plazo produce un aumento de síntomas bronquiales en niños asmáticos e irritación ocular (OMS, 2006).

En muchos países en transición y en desarrollo, no solo están ascendiendo las concentraciones de $\mathrm{SO}_{2}$ y $\mathrm{O}_{3}$ a causa del incremento del consumo de combustibles fósiles, sino que también lo hacen las de $\mathrm{NOx} \mathrm{y} \mathrm{O}_{3}$, 
Tabla 1. Fuentes habituales de contaminación del aire y contaminantes que producen (OPS, 2000).

\begin{tabular}{|c|c|c|}
\hline Categoría de la fuente & Fuente & Contaminantes emitidos \\
\hline Agricultura & Fuegos al aire libre & PSS, CO, COV \\
\hline Minería y canteras & $\begin{array}{l}\text { Minas de carbón } \\
\text { Petróleo crudo y gas natural } \\
\text { Minería de minerales no ferrosos } \\
\text { Extracción de piedra }\end{array}$ & $\begin{array}{l}\mathrm{PSS}, \mathrm{SO}_{2}, \mathrm{NOX}, \mathrm{COV} \\
\mathrm{SO}_{2}, \mathrm{PSS}, \mathrm{Pb} \\
\text { PSS }\end{array}$ \\
\hline Manufacturas & $\begin{array}{l}\text { Alimentos, bebidas, tabaco, textiles, cuero } \\
\text { Productos de la madera } \\
\text { Productos del papel, imprentas }\end{array}$ & $\begin{array}{l}\text { PSS, CO, COV, } \mathrm{H}_{2} \mathrm{~S} \\
\text { PSS, COV } \\
\text { PSS, COV } \\
\text { SPP, } \mathrm{SO}_{2}, \mathrm{CO}, \mathrm{COV}, \mathrm{H}_{2} \mathrm{~S}, \mathrm{R}-\mathrm{SH}\end{array}$ \\
\hline Industrias químicas & $\begin{array}{l}\text { Anhídrido ftálico } \\
\text { Álcalis clorados } \\
\text { Ácido clorhídrico } \\
\text { Ácido sulfúrico } \\
\text { Ácido nítrico } \\
\text { Ácido fosfórico } \\
\text { Óxido de plomo y pigmentos } \\
\text { Amoníaco } \\
\text { Carbonato de sodio } \\
\text { Carburo de calcio } \\
\text { Acido atípico } \\
\text { Alquiles de plomo } \\
\text { Anhídrido maléico, ácido tereftálico } \\
\text { Fertilizantes y plaguicidas } \\
\text { Nitrato de amonio } \\
\text { Sulfato e amonio } \\
\text { Resinas sintéticas, plásticos, fibras } \\
\text { Pinturas, barnices, lacas }\end{array}$ & $\begin{array}{l}\mathrm{PSS}, \mathrm{SO}_{2}, \mathrm{CO}, \mathrm{COV} \\
\mathrm{Cl}_{2} \\
\mathrm{HCl} \\
\mathrm{HF} \mathrm{SiF}_{4} \\
\mathrm{SO}_{2}, \mathrm{SO}_{3} \\
\mathrm{NOX} \\
\mathrm{PSS}, \mathrm{F}_{2} \\
\mathrm{PSS}, \mathrm{Pb} \\
\mathrm{PSS}, \mathrm{SO}_{2}, \mathrm{NOX}, \mathrm{CO}, \mathrm{COV}, \mathrm{NH}_{3} \\
\mathrm{PSS} \\
\mathrm{PSS}, \mathrm{NOX}, \mathrm{CO}, \mathrm{VOC} \\
\mathrm{Pb} \\
\mathrm{CO}, \mathrm{VOC} \\
\mathrm{PSS}, \mathrm{NH}_{3} \\
\mathrm{PSS}, \mathrm{NH}_{3}, \mathrm{HNO}_{3} \\
\mathrm{COV} \\
\mathrm{PSS}, \mathrm{COV}, \mathrm{H}_{2} \mathrm{~S}, \mathrm{CS}_{2} \\
\mathrm{PSS}, \mathrm{COV}\end{array}$ \\
\hline $\begin{array}{l}\text { Manufactura de sustancias } \\
\text { químicas }\end{array}$ & $\begin{array}{l}\text { Jabón } \\
\text { Negro carbón, tinta de imprenta } \\
\text { Trinitrotolueno }\end{array}$ & $\begin{array}{l}\text { PSS } \\
\text { PSS, } \mathrm{SO}_{2}, \mathrm{NOX}, \mathrm{CO}, \mathrm{COV}, \mathrm{H}_{2} \mathrm{~S} \\
\mathrm{PSS}, \mathrm{SO}_{2}, \mathrm{NOX}, \mathrm{SO}_{3}, \mathrm{HNO}_{3}\end{array}$ \\
\hline Refinerías de petróleo & Distintos productos del petróleo y carbón & $\mathrm{PSS}, \mathrm{SO}_{2}, \mathrm{NOX}, \mathrm{CO}, \mathrm{COV}$ \\
\hline $\begin{array}{l}\text { Manufactura de productos } \\
\text { minerales no metálicos }\end{array}$ & $\begin{array}{l}\text { Vidrio y cristal } \\
\text { Derivados estructurales de la arcilla } \\
\text { Cemento, yeso, cal }\end{array}$ & $\begin{array}{l}\mathrm{PSS}, \mathrm{SO}_{2}, \mathrm{NOX}, \mathrm{CO}, \mathrm{COV}, \mathrm{F} \\
\mathrm{PSS}, \mathrm{SO}_{2}, \mathrm{NOX}, \mathrm{CO}, \mathrm{COV}, \mathrm{F}_{2} \\
\mathrm{PSS}, \mathrm{SO}_{2}, \mathrm{NOX}, \mathrm{CO}\end{array}$ \\
\hline Industrias básicas del metal & $\begin{array}{l}\text { Hierro y acero } \\
\text { Metales no ferrosos }\end{array}$ & $\begin{array}{l}\mathrm{PSS}, \mathrm{SO}_{2}, \mathrm{NOX}, \mathrm{CO}, \mathrm{COV}, \mathrm{Pb} \\
\mathrm{PSS}, \mathrm{SO}_{2}, \mathrm{~F}, \mathrm{~Pb}\end{array}$ \\
\hline Generación de energía & Electricidad, gas, vapor & $\mathrm{PSS}, \mathrm{SO}_{2}, \mathrm{NOX}, \mathrm{CO}, \mathrm{COV}, \mathrm{SO}_{3}, \mathrm{~Pb}$ \\
\hline Comercio minorista del petróleo & Depósitos de combustible, operaciones de llenado & cov \\
\hline Transporte & Motores de combustión & $\mathrm{PSS}, \mathrm{SO}_{2}, \mathrm{NOX}, \mathrm{CO}, \mathrm{COV}, \mathrm{Pb}$ \\
\hline Servicios comunitarios & Incineradores municipales & $\mathrm{PSS}, \mathrm{SO}_{2}, \mathrm{NOX}, \mathrm{CO}, \mathrm{COV}, \mathrm{Pb}$ \\
\hline
\end{tabular}


debido al aumento de las emisiones provenientes del tráfico y de las emisiones industriales de HC (OPS, 2000).

Alrededor de las fuentes de origen, se producen a menudo problemas muy graves de contaminación del aire de diferente tipo. En las zonas en que las circunstancias topográficas reducen su dispersión atmosférica puede haber, períodos de tiempo con concentraciones peligrosamente altas. En ciertas condiciones climáticas, tales como la inversión térmica y los vientos de poca velocidad, las concentraciones se pueden mantener altas durante varios días (Medeiros $\varepsilon$ Gouveia, 2005).

\section{CALIDAD DEL AIRE Y FACTORES CLIMATOLÓGICOS}

Se deben tener en cuenta la contaminación del aire intra y extradomiciliario, las deficientes condiciones de vivienda, el hacinamiento, el cocinar con material de biomasa, madre o cuidador fumador que convierten a los niños en fumadores pasivos. La importancia de una calidad del aire adecuada en la perspectiva de crear escenarios favorables a la salud es evidente (Cazzola et al. 2006).

En el mundo varios grupos han evaluado el impacto de la contaminación del aire en la enfermedad respiratoria aguda y recurrente de los niños y niñas. Los hallazgos muestran una asociación significativa, entre la incidencia de tos y síntomas de tracto respiratorio superior y la contaminación del aire (Lee et al. 2007). Asociaciones semejantes, se han encontrado en estudios realizados en Colombia.

La contaminación del aire vincula dos escenarios complementarios: de un lado, los aspectos relacionados con el aire exterior, que incorpora en su análisis los aspectos climatológicos y, de otro, los contaminantes generados por agentes químicos o partículas, cuyo origen deriva de fuentes fijas o móviles (Bringgs et al. 2000).

Dentro de los factores climatológicos evaluados cabe mencionar que existen áreas en Bogotá donde la capacidad depuradora de las lluvias es menor, particularmente en las localidades de Ciudad Bolívar, Usme y Tunjuelito. Tal fenómeno, aunado al desarrollo de la actividad minera en el área, la deficiencia de las vías, el mal estado del transporte y la escasa circulación del viento, hacen del área suroriental de la ciudad un espacio crítico para la presentación de sintomatología respiratoria (Secretaría Distrital de Salud, 2004).

Otra variable a considerar es la temperatura. El fenómeno de inversión térmica se prolonga en los periodos más fríos y de mayor pluviosidad, favoreciendo la permanencia de partículas contaminantes en el ambiente, lo cual, aumenta el periodo de exposición a estas (Ebi $\&$ Paulson, 2007).

Factores Atmosféricos: De manera complementaria, además de estas variables de carácter meteorológico es necesario considerar los principales contaminantes atmosféricos, entre los que se consideran el material particulado menor a 10 micras, los óxidos de azufre, de nitrógeno y el $\mathrm{O}_{3}$. Cabe anotar que las principales fuentes de $\mathrm{SO}_{2}$ y CO son las industrias y el transporte, respectivamente. En nuestro medio, las localidades donde la densidad de las partículas contaminantes es mayor en la ciudad de Bogotá son: Ciudad Bolívar, Tunjuelito, Kennedy, Engativá, Suba, Fontibón y en algunas áreas de Puente Aranda.

Contaminación ambiental intra y extradomiciliaria: La contaminación del aire interior tiene mil veces más posibilidades de alcanzar los pulmones que ese mismo contamínate liberado al espacio exterior. Según la OMS, en los países en desarrollo la fuente más importante de contaminación es el uso doméstico de combustibles de biomasa y carbón para la cocina, y el hábito del tabaquismo intradomiciliario (Chen et al. 2007). Estudio, como el de Solarte et al. (2006), evidenciaron tabaquismo pasivo en niños y exposición a contaminación intradomiciliaria en casi $30 \%$ de los hogares de las localidades de Tunjuelito y Engativá.

Riesgo a nivel pulmonar: Los principales efectos nocivos en el aparato respiratorio asociados con contaminación intra y extradomiciliaria son alteración estructural e inhibición del sistema mucociliar, hiperplasia celular epitelial, inflamación de la mucosa respiratoria con hipersecreción y aumento de la permeabilidad, bronco constricción refleja y aumento de la reactividad bronquial en general e inhibición de macrófagos y alteración de la respuesta inmune (Chen et al. 2007). 


\section{EFECTOS SOBRE GRUPOS POBLACIONALES}

\section{Efectos de la polución en niños y niñas menores de cinco años}

En Latinoamérica por cada cien consultas pediátricas ambulatorias, aproximadamente 70 se deben a la IRA; de igual manera, se reporta que este evento contribuye con una de cada dos hospitalizaciones en servicios pediátricos, principalmente en épocas de invierno. Entre los pacientes que requieren hospitalización, entre 30 y $60 \%$ presentan neumonía y asma (Magas et al. 2007).

En Colombia, las infecciones respiratorias agudas continúan ocupando los primeros lugares de morbilidad y de mortalidad en el grupo de niños y niñas menores de cinco años. Dentro de las infecciones respiratorias agudas, la bronquiolitis, la bronconeumonía y la neumonía son las responsables de casi todas las muertes evitables para este grupo etáreo, especialmente en los grupos poblacionales más vulnerables (Tafur et al. 1997).

En México donde persiste una alta contaminación, se describen entre el 8,3 -12,7\% de consultas de urgencias del tracto respiratorio alto y bajo; en Latinoamérica mueren al año más de 500 mil niños, de ellos, entre 100 mil y 150 mil lo hacen por neumonía (Hernández et al. 2007). En Colombia mueren diariamente cerca de 48 niños por enfermedades prevenibles o fácilmente curables; de ellos $10 \%$ mueren por neumonía y un alto porcentaje de éstos son lactantes (Rubio \& Sánchez, 1999). Si bien en Bogotá durante el periodo 2000-2003 la tasa de mortalidad por neumonía se redujo en cerca de 50\% (pasando de 39,1 a 20,2 por 100.000 menores de cinco años), esta reducción no ha sido homogénea en las diferentes localidades. El grupo poblacional principalmente afectado lo constituyen los menores de dos años, pues durante el mismo periodo 2000-2003, $80 \%$ de las muertes ocurrieron en este grupo. Durante el primer semestre de 2004, se presentaron 162 casos de muerte por neumonía, de los cuales 78,8\% (127 casos) fueron los niños residentes en Bogotá y 21,6\% (35 casos) corresponden a niños procedentes de otros lugares. En el primer semestre de 2003, se registraron 88 muertes por neumonía de niños residentes en Bogotá, lo cual significa, respecto del primer semestre de 2004, un incremento de 44,3\% (Secretaría Distrital de Salud, 2004). Estos datos contrastan con los observados en población mexicana donde no solo hay un compromiso respiratorio sino cardiaco y cerebral (Calderón et al. 2007). En Bogotá, las épocas del año con mayor riesgo de enfermar y morir por dolencia respiratoria son aquellas de mayor pluviosidad.

Factores de riesgo. Múltiples factores subyacen y se relacionan con la enfermedad respiratoria aguda:

Factores socioeconómicos: incluyen el bajo nivel de ingresos, el desempleo, el desplazamiento, la pobreza y el bajo nivel de escolaridad. Es claro que la pobreza, el detrimento de las condiciones socioeconómicas de las personas y el trabajo informal aumentan la vulnerabilidad de la población menor de cinco años y la de mayor de 60 años a la enfermedad respiratoria aguda (Harari $\mathcal{E}$ Harari, 2006).

Factores biológicos: madre adolescente, esquema incompleto de vacunación en el niño o niña. Alteraciones genéticas, aumento de los episodios asmáticos (Gauderman et al. 2007).

Factor nutricional: incluye el antecedente de prematurez, el bajo peso al nacer y la desnutrición del niño o niña. A propósito de lo anterior, con respecto a la situación nutricional, durante 2003, se estableció que del total de nacidos vivos en Bogotá, 11\% presentaban bajo peso al nacer, en tanto que la prevalencia de bajo peso al nacer en el total de nacidos a término y en parto simple fue de 6,3\% y el déficit de peso fue de 33.8\% (Secretaría Distrital de Salud, 2004).

Prácticas alimentarías: carencia de lactancia materna o menor duración de la lactancia favorece la infección respiratoria posnatal y en la infancia (Nickerson, 2006).

Carencias en los servicios de salud: barrera de acceso a los servicios y la falta de aseguramiento, carencia de programas orientados a la infancia, la no identificación temprana de signos de alarma por parte de cuidadores, entre otros, son factores predominantes en población de bajos recursos que no tiene acceso a los servicios de salud (Kjellstrom et al. 2007). En nuestro medio, se ha notado el aumento de las consultas por enfermedad respiratoria en niños en la transición de periodos secos y el lluvioso, se promueve el uso de vacuna antigripal pero no se le ha dado importancia a los efectos del medio ambiente sobre la salud pulmonar infantil. 


\section{Contaminación del aire y riesgo para la salud durante el embarazo}

Función pulmonar: La función pulmonar durante el embarazo presenta diferentes modificaciones a través de sus tres trimestres, destacándose al final un aumento del diafragma, disminución del espacio anatómico con moderada hipoventilación e hiperventilación compensatoria. Estos cambios, los cuales afectan al sistema respiratorio, destacando entre ellos la hiperventilación. Existe un aumento del volumen minuto, aumento del consumo de oxígeno, disminución del volumen de reserva espiratorio y del volumen residual, con una disminución de la capacidad residual funcional. Estos cambios son debidos a un aumento de la progesterona, la cual actúa estimulando el centro respiratorio. Las consecuencias de la hiperventilación son una reducción de la presión de $\mathrm{CO}_{2}\left(\mathrm{PaCO}_{2}\right)$ (hipocapnia), con aumento de la excreción renal de bicarbonato que determina una leve alcalosis respiratoria. $\mathrm{La}^{\mathrm{PaO}_{2}}$ en el embarazo, se encuentra elevada en respuesta a la hipocapnia (Sulavick, 1995).

La disnea puede ser un hecho fisiológico que se presenta en un $50 \%$ y $75 \%$ de las embarazadas durante el segundo y tercer trimestre, respectivamente. La disnea parece ser debida a cambios en la conformación de la pared torácica, a la posición del diafragma y a alteraciones en la sensibilidad del centro respiratorio. Además de los cambios fisiológicos descritos, la embarazada puede desarrollar enfermedades respiratorias agudas, que pueden ser causa de morbimortalidad materna y fetal (Cabello et al. 2003). Muchos de los cuadros agudos, se presentan con signos y síntomas que recuerdan los cambios propios de la gestación y, por lo tanto, pueden pasar inadvertidos. Entre estas enfermedades debe ser destacada el asma bronquial (Schatz, 1999), la neumonía (Berkowits \& La Sala, 1990; Rodríguez \& Niederman, 1992), tromboembolismo pulmonar (TEP) y la trombosis venosa profunda (TVP) (Danilenko-Dixon et al. 2001).

Factores de Riesgo: Las causas de malformaciones congénitas se han dividido en tres categorías: desconocida, genéticas y medio ambientales. La OMS estima que de la mayoría de los abortos en el primer trimestre el $60 \%$ son debidas a alteraciones cromosomicas, 3-6\% a alteraciones del desarrollo y un $3 \%$ a causas medioambientales (Jean et al. 2006). Dentro de las diferentes malformaciones descritas, se incluye paladar hendido, anencefalia, espina bifida, malformaciones cardiacas, estenosis pilóricas, hipospadias, hernia inguinal, equino varo, luxación congénita de cadera. Algunas atribuibles a factores multifactoriales (González Fernández, 1992).

Dentro de los factores de riesgo durante le embarazo, se describen los de origen materno y ambientales, como se muestra en la Tabla 2.

Indicadores: Como indicadores, se aceptan las tasas de morbimortalidad perinatal, de malformaciones y factores atribuibles a la contaminación ambiental. Los valores de los diferentes contaminantes de acuerdo a la Agencia de protección ambiental de los Estados Unidos (USEPA, 1979) (Tabla 3).

\section{Calidad del aire y efectos sobre el embarazo}

Efecto del Ozono $\left(\mathrm{O}_{3}\right)$ : La contaminación fotoquímica, se forma en el aire exterior por una serie compleja de reacciones que involucran a los óxidos de nitrógenos, composiciones orgánicas volátiles y la presencia de la luz del sol. El ozono $\left(\mathrm{O}_{3}\right)$ es un gas que existe, junto con otras moléculas de oxidantes y partículas finas, en la mezcla de contaminantes ("smog"). En muchas grandes ciudades, con tránsito pesado y combustión don diesel (Leem et al. 2006).

Tabla 2. Factores de riesgo maternos y ambientales.

\begin{tabular}{|l|l|}
\hline \multicolumn{1}{|c|}{ MATERNOS } & \multicolumn{1}{c|}{ AMBIENTALES } \\
\hline $\begin{array}{l}\text { Desnutrición } \\
\text { Falta de control prenatal }\end{array}$ & \begin{tabular}{l}
\multicolumn{1}{c|}{ Contacto con plaguicidas, fumigantes, tóxicos ambientales (humos, } \\
gases). \\
Avitaminosis
\end{tabular} \\
Anemia & Aguas contaminadas. \\
Diabetes & Intoxicación por medicamentos, cigarrillo, drogadicción. \\
\hline
\end{tabular}


Tabla 3. Valores guía recomendados por la agencia de protección ambiental (EPA) de los Estados Unidos (USEPA, 1979).

\begin{tabular}{|c|c|c|}
\hline CONTAMINANTE & NORMA DE CALIDAD $\mu \mathrm{g} / \mathrm{m}^{3}$ & PERÍODO DE MUESTREO \\
\hline \multirow{2}{*}{$\mathrm{SO}_{2}$} & 80 & Promedio anual \\
\cline { 2 - 3 } & 365 & Promedio 24 horas \\
\cline { 2 - 3 } & 1300 & Promedio 3 horas \\
\hline \multirow{2}{*}{$\mathrm{CO}$} & 10 & Promedio 8 horas \\
\cline { 2 - 3 } & 40 & Promedio 1 hora \\
\hline $\mathrm{NO}_{2} \mathrm{O}_{3}$ & 100 & Promedio anual \\
\hline \multirow{2}{*}{$\mathrm{PM}-10$} & 157 & Promedio 8 horas \\
\hline Plomo & 235 & Promedio 1 hora \\
\hline & 50 & Promedio anual \\
\cline { 2 - 3 } & 150 & Promedio 24 horas \\
\hline
\end{tabular}

La mayoría de los estudios sobre los efectos de salud del $\mathrm{O}_{3}$, se han enfocado sobre la exposición a corto plazo y han indicado una cantidad de efectos agudos del $\mathrm{O}_{3} \mathrm{y}$ otros oxidantes fotoquímicos, incluyendo consultas en el servicio de Urgencias y Hospitalización por síntomas respiratorios. En un estudio conducido en la Ciudad de México, se observa un incremento de $10 \%$ (95\% Cl 7 a $13 \%)$ para las enfermedades de vías respiratorias altas durante el invierno relacionado con un aumento de 50ppb en el máximo diario de $1 \mathrm{~h}$ de ozono (Téllez-Rojo et al. 1997).

Los cambios en funciones pulmonares, síntomas y enfermedades respiratorias han sido observados en varios estudios. Castillejos et al. (1992) reportaron un efecto agudo y un efecto sub-agudo para el $\mathrm{O}_{3}$ sobre funciones pulmonares. Un aumento de 53ppb para el promedio de niveles de $\mathrm{O}_{3}$ en $48 \mathrm{~h}$ predecía un decremento de $2 \%$ en flujo ventilación espiratoria (FEV) y un decremento del 7,4\% en el flujo basal espiratorio (FFEB). Un decremento mayor fue observado en niños con tos crónica, flema y silbidos respiratorios.

No se encontraron informes sobre efecto del $\mathrm{O}_{3}$ sobre el embarazo.

Dióxido de Azufre $\left(\mathrm{SO}_{2}\right)$ : El dióxido de azufre es un gas soluble en agua, formado de la oxidación del azufre que contamina el carbón y los combustibles de petróleo. Consecuentemente, el gas es emitido por plantas de energía potenciadas por carbón o crudo por procesos industriales que involucran la combustión de combustibles fósiles. El $\mathrm{S}_{2}$ y la contaminación por partículas son emitidos en conjunto por fuentes de combustión y existen como componentes de una mezcla compleja (Bascom et al. 1996).

Efectos sobre el embarazo: Algunos estudios recientes se han enfocado sobre el impacto de la contaminación del aire en el crecimiento del feto, el peso al nacer y otros resultados del embarazo a partir de la preocupación creciente de que la contaminación del aire puede afectar el desarrollo del feto. Relaciones significativas del tipo exposición-respuesta entre la exposición materna a $\mathrm{SO}_{2}$, amenaza de parto preterminó y bajo peso al nacer fueron observadas en estudios conducidos en China (Wang et al. 1997) y en la República Checa (Bobak, 2000). Similarmente, la exposición a PM10 ambiental ha sido relacionada al retardo del crecimiento intrauterino (RCIU) en un estudio conducido en el norte de Bohemia. Estos resultados sugieren un efecto dañino de carácter permanente dado que el bajo peso al nacer y $\mathrm{RCIU}$ ha sido asociados con la salud respiratoria en etapas posteriores de la vida (Delmeek et al. 1999). RCIU puede llevar a una susceptibilidad exagerada a la exposición a la contaminación del aire y a otros factores ambientales (Gold et al. 1999). Existe claramente la necesidad de más investigación para identificar las consecuencias de la exposición a la contaminación del aire sobre el desarrollo fetal durante el embarazo.

Monóxido de carbono (CO): Se reportan distintos efectos en la salud asociados con la exposición a CO en niños: efectos perinatales y efectos de comportamiento neurológico. El monóxido de carbono lleva a una capacidad reducida de recepción de oxígeno. Los 
síntomas clásicos de la intoxicación por CO son dolores de cabeza y mareos a niveles de Carboxi-hemoglobina (COHb), entre el 10 y el 30\% y dolor de cabeza severo, síntomas cardiovasculares y malestar a niveles arriba del $30 \%$. Por encima del $40 \%$, existe el riesgo considerable de coma y muerte (Salam et al. 2005).

La exposición a CO puede afectar el feto directamente por el déficit de oxígeno sin que los niveles de COHB en la sangre fetal se eleven. Durante la exposición a niveles altos de $\mathrm{CO}$, la hemoglobina de la madre difícilmente libera su oxígeno, con la consecuencia de un descenso en la presión del oxígeno en la placenta y, por lo tanto, en la sangre fetal. Las investigaciones se han enfocado principalmente sobre los efectos del tabaquismo durante el embarazo. Los efectos principales son: peso reducido al nacer y un desarrollo postnatal retrasado (Dugandzic et al. 2006). Limitaciones en el desarrollo del comportamiento neurológico han sido relacionadas también con el tabaquismo materno durante el embarazo. El sistema nervioso central del feto es particularmente susceptible a $\mathrm{CO}$, mencionándose que algunos retrasos del desarrollo intelectual y neurológico, posiblemente de largo plazo, se puedan derivar del tabaquismo materno durante el embarazo (USEPA, 1979). En nuestro medio, no se ha estudiado el efecto de la contaminación ambiental y embarazo, solo se anotan las consultas por faringe-amigdalitis, asma y rinitis, pero sin correlación con el medio ambiente.

\section{ENFERMEDADES RESPIRATORIAS EN ADULTOS MAYORES DE 60 AÑOS}

Contaminación ambiental y su efecto a nivel respiratorio. La contaminación del aire en las grandes urbes impacta en forma directa la contaminación atmosférica que, por ende, incrementa la morbilidad de enfermedad respiratoria en el adulto mayor que, de hecho, presenta un moderado estado de inmunosupresión (Cakmak et al. 2007).

Algunos de los efectos en la salud atribuibles a la exposición de la contaminación atmosférica incluyen la mortalidad excesiva, por causas respiratorias, la exacerbación de asma, la disminución de la función pulmonar y las alteraciones inmunológicas (Viegi et al. 2006).

Entre las afecciones respiratorias, se encuentran los siguientes grupos:
- Infecciones agudas de las vías respiratorias superiores

- Influenza [gripe]

- Neumonía y otras infecciones agudas de las vías respiratorias inferiores

- Enfermedades crónicas de las vías respiratorias inferiores

- Enfermedades del pulmón debidas a agentes externos

- Otras enfermedades respiratorias que afectan principalmente el intersticio

- Afecciones supurativas y necróticas de las vías respiratorias inferiores

- Enfermedades de la pleura

- Neumonía en enfermedades

- Derrame pleural en afecciones

- Trastornos respiratorios en enfermedades pulmonares

- Infecciones agudas de las vías respiratorias superiores

- La enfermedad pulmonar obstructiva crónica (EPOC), con exacerbación aguda, la más frecuente en el adulto mayor (20\%) (Fauci, 1991).

Enfermedad pulmonar obstructiva crónica (EPOC): Se define como la obstrucción persistente de las vías respiratorias y puede aparecer en forma de dos trastornos diferentes: el enfisema y la bronquitis crónica. Ambas enfermedades, suelen presentarse simultáneamente o bien una acaba provocando la otra, por lo cual, enfisema y bronquitis crónica, se agrupan bajo la denominación enfermedad pulmonar obstructiva crónica y se reflejan en las dos formas citadas. Los afectados por la enfermedad pulmonar obstructiva crónica están más expuestos al riesgo de desarrollar un cáncer de pulmón que cualquier otra persona y es causa del desarrollo de cor pulmonal, por lo tanto, es susceptible de exacerbación de los síntomas ante la contaminación ambiental.

Son factores de riesgo para EPOC:

- El hábito de fumar: el cigarrillo utilizado por largos períodos inhibe el movimiento ciliar, así como la función de los macrófagos alveolares y conduce a hipertrofia e hiperplasia de las glándulas mucosecretoras.

- La contaminación atmosférica: es mayor en las grandes urbes donde la prevalencia e incidencia 
de bronquitis crónica y de enfisema con mayores. La exacerbación de la EPOC correlaciona con contaminantes, como el dióxido de azufre (Amdur, 1996) y el dióxido de nitrógeno.

- Contaminación en el ambiente laboral: como exposición al humo de leña, empresas procesadoras de plásticos, trabajadores en textileras.

- Infección sobre agregada: aumenta la morbilidad del EPOC.

- Factores genéticos: individuos homocigotos para los genes ZZ y SS tienen valores de alfa1 antitripsina inferiores a $0,5 \mathrm{~g} / \mathrm{L}$ desarrollando enfisema panacinar severo en la tercera o cuarta décadas de la vida (Kunisaki et al. 2007).

Efectos de contaminantes ambientales sobre la función pulmonar: En los últimos años se hicieron muchos estudios en las ciudades de los países desarrollados sobre las consecuencias de la contaminación del aire por PSS. Estos ensayos revelan con una constancia notable, la relación existente entre el cambio diario del número de partículas en suspensión en el aire ambiental y la variación de la mortalidad diaria. Alrededor de tres millones de muertes anuales, se deben a la contaminación del aire, constituyendo el $6 \%$ de los 50 millones totales de muertes que se producen cada año en el mundo. Muchas de éstas, se deben a las infecciones respiratorias agudas (IRA) de los niños y niñas, aunque también contribuyen a ellas las enfermedades cardiovasculares, el cáncer de pulmón y los trastornos respiratorios crónicos de los adultos (Gilliland et al. 2005). Puesto que existen interacciones con otros factores de riesgo en todas estas enfermedades, la disminución del número de muertes observada tras la reducción de un factor podría reducir la cifra de las atribuibles a la contaminación del aire y viceversa. Sin embargo, los cálculos de los estudios ofrecen ciertas dudas, ya que se han obtenido con métodos distintos, presentando resultados variables (Ulirsch et al. 2007). La experiencia a nivel hospitalario en nuestro medio es que $30-40 \%$ de las camas son ocupadas por EPOC.

Riesgos para la salud causados por la contaminación del aire por ozono: $\mathrm{El}_{3}$ y otros oxidantes fotoquímicos, se forman por la acción de la radiación solar de onda corta sobre el $\mathrm{NO}_{2}$. Los efectos de los distintos niveles de exposición al $\mathrm{O}_{3}$, se producen como un continuo, desde los síntomas respiratorios hasta los cambios de la función pulmonar y la inflamación de las vías respiratorias. La exposición al $\mathrm{O}_{3}$, se ha asociado también al aumento de los ingresos hospitalarios por trastornos respiratorios, como la exacerbación del asma (Kim et al. 2007). El efecto que tiene en la salud de una persona un determinado nivel de concentración depende del tiempo que pase al aire libre y de grado de actividad (Lagorio et al. 2006).

Ozono: Algunos mecanismos de daño señalan que el ozono altera las funciones de los macrófagos, lo cual podría estar aumentando la susceptibilidad a las infecciones respiratorias. Las respuestas agudas en los niños incluyen: aumento en los síntomas respiratorios, tasas reducidas de flujo respiratorio y reducción en la capacidad vital forzada (FVC). Los síntomas más comunes incluyen irritación en los ojos, nariz y garganta y dificultades en la inhalación profunda que empieza con un promedio de concentraciones de los oxidantes de $200 \mu \mathrm{g} /$ $\mathrm{m}^{3}$ (100ppb) por hora (Kim et al. 2007). Estas respuestas son acompañadas frecuentemente por un incremento de la resistencia en las vías respiratorias y taquipnea (ritmo de respiración acelerada). Los asmáticos tienen síntomas similares a los no asmáticos, pero también reportan una incidencia más alta de silbidos respiratorios que obligan a consumir medicamentos y a experimentar una disminución en la tasa de flujo respiratorio máximo (Avol et al. 1990).

Riesgos para la salud causados por la contaminación del aire por $\mathrm{NO}_{2}$ : En el caso de la exposición al $\mathrm{NO}_{2}$, no existen pruebas claras que demuestren una relación definida entre la concentración y la respuesta. Durante la exposición aguda, las personas sanas solo se afectan con concentraciones muy altas. Los asmáticos y los que padecen enfermedad pulmonar obstructiva crónica son más propensos a sufrir cambios agudos de la función pulmonar y de la respuesta de las vías aéreas y síntomas respiratorios. Los estudios epidemiológicos sobre la exposición crónica sugieren un abanico de efectos y genera visitas frecuentes en servicio de urgencias. El incremento de $30 \mathrm{~g} / \mathrm{m}^{3}$ en promedio diario de PM10, se relaciona con un $18,4 \%$ de muertes diarias causada por EPOC, fuera de unidades médicas (Sarnat et al. 2006).

Riesgos para la salud causados por la contaminación del aire por $\mathrm{SO}_{2}$ : La exposición extendida y repetida a $\mathrm{SO}_{2}$ aumenta el riesgo de enfermedad crónica por causas respiratorias. Los estudios epidemiológicos actuales no 
pueden distinguir entre PM 2,5 y sulfato, pero ambos son buenos sustitutos de la contaminación del aire relacionado con la combustión (Sarnat et al. 2006).

Los efectos agudos del $\mathrm{SO}_{2}$ constituyen en cambios de la función pulmonar, aumento de la resistencia específica de las vías aéreas y síntomas, tales como sibilancias y disnea. Los efectos a largo plazo resultan más difíciles de comprobar, pues la exposición al $\mathrm{SO}_{2}$ suele combinarse con la debida a las PSS.

Riesgos para la salud causados por la contaminación del aire por CO: El CO se combina con la hemoglobina para formar carboxihemoglobina, que reduce la capacidad de transporte de oxígeno de la sangre. Su unión con otras hemoproteínas influye directamente en la función de los órganos afectados, como el encéfalo y el aparato cardiovascular y en el feto en desarrollo. Las consecuencias neuroconductuales del CO son, con ciertos niveles de exposición, alteración de la capacidad de concentración y del rendimiento cognitivo. La hemoglobina fetal tiene mayor afinidad por el CO, lo que puede influir en el peso al nacer (OPS, 2000,). Por otra parte, se ha asociado las partículas PM lesiones a nivel vascular y trombosis en población adulta (Sullivan et al. 2007).

Se conocen efectos nocivos para la salud de numerosos contaminantes inorgánicos, como el arsénico, el cadmio, el plomo, el mercurio, el manganeso y el níquel; también se han estudiado los de los contaminantes orgánicos volátiles. En realidad, las personas están expuestas a mezclas diversas de contaminantes aéreos con efectos aditivos, sinérgicos o antagonistas que de alguna manera favorecerían el riesgo de cáncer pulmonar (Ranmacunar et al. 2007).

La mayoría de los estudios sobre los efectos de salud del $\mathrm{O}_{3}$, se han enfocado sobre la exposición a corto plazo y han indicado una cantidad de efectos agudos del $\mathrm{O}_{3}$ y otros oxidantes fotoquímicos, incluyendo visitas a la sala de emergencia y admisión hospitalaria, síntomas respiratorios y el cambio en los parámetros de función pulmonar (Romieu et al. 2004). El incremento de los niveles de ozono más allá de las normas establecidas, aumenta el total de muertes debidas a EPOC.

La exposición elevada puede ocasionar una intoxicación aguda; coma y colapso empiezan a ocurrir a niveles de carboxihemoglobina $(\mathrm{COHB})>40 \%$. No obstante, la mayoría de las exposiciones a $\mathrm{CO}$ en escenarios urbanos son inferiores en varios grados de magnitud a los asociados con la intoxicación y el envenenamiento (Prieto et al. 2007). Es de menor relevancia para el desarrollo de EPOC.

\section{RECOMENDACIONES}

De acuerdo al Boletín No. 22 de la Secretaría de Salud Distrital (Solarte et al. 2006), los principales contaminantes que se respiran en Bogotá están compuestos por material particulado (MP), los óxidos de azufre (SOx), los óxidos de nitrógeno (NOx), el monóxido de carbono (CO) y el ozono $\left(\mathrm{O}_{3}\right)$. Las mayores emisiones de MP, NOx y CO son causadas por fuentes móviles, como transporte público y particular. Las de SOx son fijas, como fábricas y chimeneas. Existen, además, los humos provenientes de las estufas de carbón, de la cocción de los alimentos, del cigarrillo, del polvo resuspendido del suelo y los humos de otras viviendas.

- Estar atentos a niveles de contaminación, sitio de residencia y población vulnerable, como niños menores de doce años, antecedentes de asma, síndrome bronco obstructivo recurrente y los mayores de 60 años.

- Recomendaciones diarias de auto cuidado, cambios de temperatura y eliminar los elementos contaminantes locales.

- Recomendaciones nutricionales, líquidos, verduras y frutas ricas en vitamina $C$.

- Evitar el ejercicio al aire libre de personas con antecedentes cardiacos o respiratorios de niños y de ancianos.

- Disminuir los contaminantes intra y extra domiciliarios.

- Acatar las disposiciones de las autoridades con relación a la contaminación ambiental.

- Asistir a las campañas de concientización de la población sobre los efectos de la contaminación ambiental.

- Acudir al centro médico más cercano ante el menor síntoma respiratorio sobre todo en la población vulnerable.

- Continuar, a través del Ministerio de Vivienda y Medio ambiente, secretarías de salud, entes territoriales, universidades y centros dedicados a la preservación 
del medio ambiente, con la creación de políticas y acciones tendientes a prevenir el deterioro atmosférico $y$, en esas condiciones, preservar la salud humana.

- Consultar la red de monitoreo del aire, las cuales se publican diariamente por el Departamento Administrativo del Medio Ambiente (DAMA, 2005) que permite conocer el grado de contaminación de su barrio.

\section{BIBLIOGRAFÍA}

AMDUR, M. 1996. Particles in our air: concentrations and health effects. In: Wilson, R.; Spengler, J. eds. Animal Toxicology. Harvard Universitity Press. p.85-112.

AVOL, E.L.; LINN, W.S.; SHAMOO, D.A.; ANDERSON, K.R.; PENG, R.C.; HACKNEY, J.D. 1990. Respiratory responses of young asthmatic volunteers in controlled exposures to sulfuric acid aerosol. Am. Rev. Respir. Dis. 142:343-348.

BASCOM, R.; BROMBERG, P.; COSTA, D.; KESAVANATHAN, J.; SWIFT, D.L.; FITZGERALD, T.K.; PERMUTT, T. 1996. Health effects of outdoor air pollution. Am. J. Respir. Crit. Care Med. 153:477498.

BERKOWITS, K.; LA SALA, A. 1990. Risk Factors associated with the increasing prevalence of pneumonia during pregnancy. Am. J. Obstet. Gynecol. 163:981-985.

BOBAK, M. 2000. Outdoor air pollution, low birth weight, and prematurity. Environ. Health Perspec. 2:173-176.

BRINGGS, D.; DE HOOGH, C.; GULLIVER, L.; WILLS, J.; ELLIOTT, P.; KINGHAM, S.; SMALLBONE, K. 2000. A regression-based method for mapping traffic-related air pollution: application and testing in four contrasting urban environments. Sci. Total Environ. 253:151-167.

CABELLO, H.A.; MANIEU, D.M.; RUIZ, M.C. 2003. Enfermedades respiratorias y embarazo. Rev. Chil. Enf. Respir. 19:160-165.
CAKMAK, S.; DALES, R.; VIDAL, C.B. 2007. Air pollution and mortality in Chile: susceptibility among the elderly. Environ. Health Perspect.115:524-527.

CALDERÓN, G.I.; FRANCO, L.M.; TORRES, J.R.; HENRIQUEZ, R.C.; BARRAGÁN, M.G.; VALENCIA, S.G.; CONZÁLEZ, M.A.; REYNOSO, R.R.; VILLARREAL, C.R.; REED, W. 2007. Pediatric respiratory and systemic effects of chronic air pollution exposure:nose,lung,hearth and brain pathology. Toxicol. Pathol. 35:154-162.

CASTILLEJOS, M.; GOLD, D.; DOCKERY, D.; TOSTESON, T.; BAUM, T.; SPEIZER, F. 1992. Effects of ambient ozone on respiratory function and symptoms in Mexico City Schoolchildren. Am. Rev. Respir. Dis. 145:276-282.

CAZZOLA, M.; DONNER, C.F.; HANANIA, N.A. 2006. One hundred years of chronic obstructive pulmonary disease (COPD). Respir. Med. 101:1049-1065.

CEPIS-OPS. 2006. Curso de autoinstrucción de evaluación del riesgo asociado a contaminación del aire. Disponible desde Internet en: http://www. cepis.ops-oms.org/bvsci/E/fulltext/riesgo/lecciones/leccion2d. (con acceso 09/12/06).

CHEN, T.M.; GOKHALE, J.; SHOFER, S.; KUSCHENER, W.G. 2007. Outdoor air pollution: ozone health effects. Am. J. Med. Sci. 4:244-248.

DANILENKO-DIXON, D.R.; HEIT, J.A.; SILVERSTEIN, M.D.; YAWN, B.P.; PETTERSON, T.M.; LOHSE, C.M. 2001. Risk Factors for deep vein thrombosis and pulmonary embolism during pregnancy or post partum: A population-based, case-control study. Am. J. Obstet. Gynecol. 184:104-110.

DELMEEK, J.; SELEVAN, S.G.; BENES, I.; SOLANSKY, I.; SRAM, R.J. 1999. Fetal growth and maternal exposure to particulate matter during pregnancy. Environ. Health Perspect. 107:475-480.

DEPARTAMENTO TÉCNICO ADMINISTRATIVO DEL MEDIO AMBIENTE DAMA. 2005. Subdirección Ambiental Sectorial. Red de Monitoreo de Calidad del Aire de Bogotá D.C. Informe Mensual de calidad del aire de Bogotá. Enero. 60p. 
DOCKERY, D.W. 1994. Epidemiologic evidence of cardiovascular effects of particulate air pollution. Environ. Health Perspect. 109 Suppl 4:483-486.

DUGANDZIC, R.; DODDS, L.; STIEB, D. 2006. The association between low level exposures to ambient air pollution and term low birth weight: a retrospective cohort study. Environ Health. 5:3-10.

EBI, K.L.; PAULSON, J.A. 2007.Climate change and children. Pediatr. Clin. North Am. 2:213-226.

FAUCI, A.S. 1991. Enfermedades del Aparato Respiratorio. En: Isselbacher, K.J.; Braunwald, E.; Wilson, J.D. eds HARRISON'S. Principles of Internal Medicine, Ed. Interamericana-McGraw Hill (New York). p.1074-1090.

FONDO FINANCIERO DISTRITAL DE SALUD, CENTRO DE ESTUDIOS PARA LA PREVENCION DE DESASTRES (CEPREVE). 1999. Identificación de los riesgos y amenazas de origen antrópico de las localidades de Bogotá. Tercer Mundo. Ed. S.A. Bogotá. Colombia. 65p.

GAUDERMAN, W.J.; VORA, H.; MCCONNELL, R.; BERHANE, K.; GILLILAND, F.; THOMAS, D.; LURMANN, F.; AVOLE-KUNZLI, N.; JERRET, M.; PETRES, J. 2007. Effect of exposure to traffic on lung development from 10-18 years of age: a cohort study. Lancet. 369:571-577.

GILLILAND, F.; AVOL, E.; KINNEY, P.; JERRET, M.; DVONCH, T.; LURMANN, F.; BUCKLEY, T.; BREYSSE, P.; KELLER, G.; VILLIER, T.; McCONMELL, R. 2005. Air pollution exposure assessment for epideoloig studies of pregnant women and children: Lessons learned from the Centers for Children's environmental health and disease prevention research. Environ. Health Perspect. 113:1447-1454.

GOLD, D.R.; BURGE, H.A.; CAREY, V.; MILTON, D.K.; PLATTS-MILLS, T.; WEISS, S.T. 1999. Predictors of repeated wheeze in the first year of life: the relative roles of cockroach, birth weight, acute lower respiratory illness, and maternal smoking. Am. J. Resp. Crit. Care Med. 160:227-236.
GONZÁLEZ FERNÁNDEZ, E. 1992. Toxicocinética y evaluación de riesgos para la salud producidos por la exposición a los compuestos de cromo. Medicina y Seguridad en el Trabajo. Hum. Toxicol. 10:34-36.

HARARI, R.; HARARI, H. 2006. Children's environment and health in Latin America: the Ecuadorian case. Ann. N Y Acad. Sci. 1076: 660-677.

HEINRICH, J.; HOELSCHER, B.; WJST, M. 1999. Respiratory diseases and allergies in two polluted areas in East Germany. Environ. Health Perspect. 107:53-62.

HERNÁNDEZ-CADENA, I.; ARRAZA-VILLAREAL, A.; RAMÍREZ-AGUILAR, M.; MORENO-MACÍAS, H.; MULLER, P.; CARBAJAL-ARROYO, L.A.; ROMIE, I. 2007. Infant morbidity caused by respiratory disease and its relation with the air pollution in Juarez city, Chihuahua, Mexico. Salud Publica Mex. 49:27-36.

JEAN, D.B.; ZHAN, F.B.; SUÁREZ, L.; LANGLOIS, P.H.; MOODY, K. 2006. Linking environmental hazards and birth defects data. I J Occup. Environ Health. 12:126-33.

KETTUNEN, J.; LANKI, T.; TIITANEN, P.; AALTO, P.P.; KOSKENTALO, T.; KULMALA, M.; SALOMAA, V.; PEKKANEN, J. 2007. Associations of fine and ultrafine particulate air pollution with stroke mortality in an area of low air pollution levels. Stroke 38:918-922.

KIM, D.H.; KIM, Y.S.; PARK, J.S.; KWON, H.J.; LEE, K.Y.; LEE, S.R.; JEE, Y.K. 2007. The effects of on-site measured ozone concentration on pulmonary function and symptoms of asthmatics. J. Korean Med. Sci. 22:30-36.

KJELLSTROM, T.; FRIEL, S.; DIXON, J.; CORVALAN, C.; REHFUESS, E.; CAMPBELL-LENDRUM, D.; GORE, F.; BARTRAM, J. 2007. Urban Environmental Health Hazards and Health Equito. J. Urban Health. 84:376-381

KREWKI, D.; RAINHAM, D. 2007. Ambient air pollution and population health: overview. J. Toxicol. Environ. Health 70:275-283. 
KUNISAKI, K.M.; RICE, K.L.; NIEWOEHNER, D.E. 2007. Management of acute exacerbations of chronic obstructive pulmonary disease in the elderly: an appraisal of published evidence. Drugs Aging. 24:303-324.

LAGORIO, S.; FORASTIERE, F.; PISTELLI, R.; IAVARONE, I.; MICHELOZZI, P.; FANO, V.; MARCONI, A.; ZIEMACKI, G.; OSTRO, B.D. 2006. Air pollution and lung function among susceptible adult subjects: a panel study. Environ. Health. 5:5-11.

LEE, I.M.; TSAI, S.S.; CHANG, C.C.; HO, C.K.; YANG, C.Y. 2007. Air pollution and hospital admissions for chronic obstructive pulmonary disease in a tropical city. Kaohsinng. Taiwan. Inh. Toxicol 19:393-398.

LEEM, J.H.; KAPLAN, B.M.; SHIM, Y. 2006. Exposure to air pollutant during pregnacy and preterm delivery. Environ. Health Perspect. 114:905-910.

MAGAS, O.K.; GUNTER, J.T.; REGENS, J.L. 2007. Ambient air pollution and daily pediatric hospitalizations for asthma. Environ. Sci. Pollut. Res. Int. 14:19-23.

MEDEIROS, A.; GOUVEIA, N. 2005. Relationship between low birth weight and air pollution in the city of Sao Paulo, Brazil. Rev. Saude Pub. 39:77-85.

NATIONAL CHILDREN`S STUDY. 2004. Study plan. Rock Ville MD. National Children Study. Disponible desde Internet en: htpp//nationalchlidrensstudy. gov (con acceso 15/06/04).

NICKERSON, K. 2006. Environmental contaminants in breast milk. J. Midwifery Womens Health. 1:26-34.

ORGANIZACIÓN MUNDIAL DE LA SALUD - OMS. 2006. Directrices sobre la calidad del aire en la protección de la salud pública. Nota descriptiva $\mathrm{N}^{\circ} 313$ - Octubre de 2006. 6p.

ORGANIZACIÓN PANAMERICANA DE LA SALUD (OPS). 2000. La salud y el ambiente en el desarrollo sostenible. Publicación científica No 572. Washington, D.C. $283 p$.

OSMOND, C.; BAKER, D.J.P. 2000. Fetal, infant and childhood growth are predictors of coronary heart disease, diabetes, and hypertension in adult men and women. Environ. Health Perspect. 18:545-553.

POPE, C.A.; BURNETT, R.T.; THUN, M.J.; CALLE, M.J. KREWISKI, D.; ITO, K. 2002. Lung cancer, cardiopulmonary mortality, and long-term exposure to fine particulate air pollution, JAMA, 287:11321141.

PRIETO, C.M.J.; MANCILLA, F.P.; ASTUDILLO, O.P.; REYES, P.A.; ROMÁN, A.O. 2007. Excess respiratory diseases in children and elderly people in a community of Santiago with high particulate air pollution. Rev Med. Chil. 135:221-228.

RANMACUNAR, A.V.; PARENT, M.E.; SIEMIATYCHI, J. 2007. Risk of lung cancer from residencial heating and cooking fuels in Montreal, Canada. Am. J. Epidemiol. 165:634-642.

RODRÍGUEZ, J.; NIEDERMAN, M. 1992. Pneumonia complicating pregnancy. Clin. Chest. Med. 4:679691.

ROMEO, E.; DeSARIO, M.; FORASTIERE, F.; COMPAGNUCCI, P.; STAFOGGIA, M.; BERGAMASCHI, A.; PERUCCICA, C.A. 2006. PM10 exposure and asthma exacerbations in pediatric age: a metaanalysis of panel and time series study. Epidemiol. Prev. 30:245-254

ROMIEU, I.; RAMÍREZ-AGUILAR, M.; MORENO-MACÍAS, H.; BARRAZA-VILLARREAL, A.; MILLER, P.; HERNÁNDEZ-CADENA, L.; CARBAJAL-ARROYO, L.A.; HERNÁNDEZ-áVILA, M. 2004. Infant mortality and air pollution: modifying effect by social class. J. Occup. Environ. Med. 46:1210-1216.

RUBIO, M.M.; SÁNCHEZ, D.A. 1999. Secretaría Distrital de Salud y Alcaldía Mayor de Bogotá. Factores de riesgo asociados a la mortalidad por neumonía en menores de un año en Bogotá. 1996-1997. Panamericana formas e impresiones. Bogotá. p.140-151.

SALAM, M.T.; MILLTEIN, J.; LI, Y.F.; LURMAN, F.W.; MARGOLIS, H.G.; GILLILAND, F.D. 2005. Birth 
outcomes and prenatal exposure to ozone, carbon monoxide and particulate matter: Results from the Children's Health Study. Environ. Heath Perspect. 113:1638-1644.

SARNAT, S.E.; COULL, B.A.; SCHWARTZ, J., GOLD, D.R.; SUH, H.H. 2006. Factors affecting the association between ambient concentrations and personal exposures to particles and gases. Environ. Health Perspect. 114:649-654.

SCHATZ, M. 1999. Asthma and pregnancy. Lancet. 353:1202-1024

SCHIKOWSKI, T.; SUGIERI, D.; RANFT, U.; GEHRING, U.; HEINRICH, J.; WICHMANN, H.E.; KRAMER, U. 2007. Does respiratory health contribute to the effects of long-term air pollution exposure on cardiovascular mortality. Respir. Res. 7:8-20

SECRETARÍA DISTRITAL DE SALUD DE BOGOTÁ (SDSB). 2004. Lineamientos técnicos y administrativos para la prevención y atención de la enfermedad respiratoria aguda. Bogotá. 59p.

SOLARTE, I.; HERNÁNDEZ, L.J.; ROJAS, N.Y. 2006. Enfermedad respiratoria aguda. Boletín ERA No 22. Secretaría de Salud. 22p.

SRAM, R.J.; BINKOBA, B.; DELMEK J. 2005. Ambient air pollution and pregnancy outcomes: a review of the literature. Environ. Health Perspect. 113:375382.

SULAVICK, S.B. 1995. Pulmonary disease. En: Burrow, G.N.; Ferris, T.F. edit Medical complications during pregnancy. W.B Saunders Co. Philadelphia USA. p.549-609.

SULLIVAN, J.H.; HUBBARD, R.; LIU, S.L.; SHEPHERD, K.; TREGA, C.A.; KOENIG, J.Q.; CHANDLER, W.I.; KAUFMAN, J.D. 2007. A community study of the effect of particulate matter on blood measures of inflammation and thrombosis in an elderly polupation. Environ. Health 6:3-11.
TAFUR, L.A.; VICTORIA, J.; SAA NAVIA, D. 1997. Características del asma bronquial en niños de Cali. Rev. Col. Médica. 28:10-15.

TÉLLEZ-ROJO, M.M.; ROMIEU, I.; PENA, M.P.; RUIZ-VELASCO, S.; MENESES-GONZÁLEZ, F.; HERNÁNDEZ-AVILA, M. 1997. Efecto de la contaminación ambiental sobre las consultas por infecciones respiratorias en niños de la Ciudad de México: Salud Pública Mex. 39:513-522.

ULIRSCH, G..; BALL, L.M.; KAYE, W.; SHY, C.M.; LEE, C.V.; CRAWFORD-BROWN, D.; SYMONS, M.; HOLLOWAY, T. 2007. Effect of particulate matter air pollution on hospital admissions and medical visits for lung and heart disease in two southeast Idaho cities. J. Expo. Sci. Environ. Epidemiol. 1:421-426.

USEPA. 1979. Revised evaluation of health effects associated with carbon monoxide exposure: an addendum to the 1979 air quality criteria document for carbon monoxide. US EPA, Research Triangle Park, NC EPA Pub No. - 600/8 83-033 .

VIEGI, G.; MAIO, S.; BALDACCI, S.; SIMONI, M.; PISTELLI, F.; CARROZZI, N. 2006. Epidemiology of the chronic obstructive pulmonary disease: environmental and occupational risk. G. Ital. Med. Lav. Ergon. 28:270-272.

WANG, X.; DING, H.; RYAN, L.; XU, X. 1997. Association between air pollution and low birth weight: A community-based study. Environ. Health Perspect. 105:514-520.

WORLD HEALTH ORGANIZATION (WHO). 1997. Air management information system (AMIS 2.0) (Program). 2.0 versión. Geneva: World Health Organization. 1p.

Recibido: Enero 19 de 2007

Aceptado: Enero 29 de 2008 